\title{
ON ASSIGNING PRAGMATIC FUNCTIONS IN ENGLISH
}

\author{
J. Lachlan Mackenzie and M. Evelien Keizer
}

\begin{abstract}
This paper presents a discussion of the treatment of the pragmatic functions Topic and Focus in Functional Grammar (Dik: 1989, ch. 13). Two questions will be addressed: (a) the theoretical question of how the interface between the static grammar (dealing with a discourse as the product of text-creating activity) and the dynamic theory of verbal interaction (dealing with discourse as the ongoing textcreating process itself) is handled with regard to pragmatic functions; and (b) the practical question whether the reader of $D i k$ (1989) finds a set of proposals that can be operationalized in the analysis of linguistic material. With regard to the former question we conclude that in the present FG treatment of Topic and Focus, the static and the dynamic approaches do not connect and that, as a result, the speaker's selection of constituents for Topic or Focus function is left unaccounted for. As for the second question, we show that Dik's proposal can be put into practice, but that the resultant analysis suffers from a number of inconsistencies and unclarities. Finally, we argue that most of these inconsistencies and unclarities can be solved if (1) we accept a different classification of Focus, and (2) assume that Topic assignment is irrelevant in English, as there is no consistent way in which Topic constituents are given special treatment, and Pl-placement can be accounted for without having to resort to Topic function.
\end{abstract}

\section{Introduction*}

With the appearance of Dik (1989), linguists have acquired a cogent and lucid presentation of the current state of Functional Grammar (FG). The book provides a broad but also detailed coverage of all fundamental aspects of the model, so that it will be of value not only to theoreticians but also to those concerned with the practical description of languages. In the present article, we have been inspired by the same duality of purpose: our argument is theoretical in nature, but is directed above all at practitioners in our ambition to test the extent to which one of the subsystems treated in Dik (1989) can be applied in the actual analysis of a language. The subsystem in question is pragmatic function

The research for this paper was conducted in the framework of research project LETT $88 / 10$ of the Free University, Amsterdam, 'Functional Language Research: Grammar and Pragmatics'. We are grateful to Machtelt Bolkestein, Chris Butler, Mike Hannay and Miriam van staden for valuable comments on an earlier version of this paper, and to an anonymous reader. 
assignment, and the language, English.

FG is conceived as being embedded within a wider pragmatic theory of verbal interaction (Dik 1989: 12). Such a theory, the outlines of which are beginning to emerge in the pragmatic literature, will be designed to account for the regularities underlying the structured, cooperative, real-time activities of language users, and will be necessarily procedural in orientation. It will concern itself with language users' plans and goals, their motivations and strategies, their problem-solving techniques, their quest for contexts and for relevance. Contributions may be expected from specialists in cognitive psychology and artificial intelligence, from anthropology and micro-sociology, from semiotics and communication theory, indeed from pragmatics in the widest sense. A theory of verbal interaction will thus be a dynamic theory, a theory of operations and processes.

A grammar, on the other hand, and FG is no exception in this respect, has a fundamentally different orientation: it seeks to describe and explain the outcome of operations and processes rather than those operations and processes themselves. It is static, not dynamic, and is essentially atemporal in its attempt to establish relationships between either virtual or attested expressions rather than follow the ongoing production or interpretation of utterances. If there appear to be certain analogies between paths through the grammar and plausible sequences of events in linguistic production, that may increase the "psychological adequacy" of the model and its "modules", but the grammar can claim no more than to be written in a "quasi-productive" mode (Dik 1989: 52). The relationship between FG and the allembracing theory of verbal interaction is thus problematic in nature, being an interface between a static and a dynamic system.

Now, of all the various subcomponents of the grammar, there are two which obviously stand at the interface with the ambient theory of verbal interaction: one is the analysis of the linguistic expression as a speech act, with an indication of illocutionary force being incorporated into the representation of a clause (Dik 1989: 248; 254 ff.); the other, to be focused on in the present article, is the assignment of pragmatic functions. The very definition of pragmatic functions, as specifying "the informational value of different parts of the clause, in relation to the speaker's estimate of the 
pragmatic information of the addressee" (Dik 1989: 60), makes it clear that they are partially concerned with a dynamic quantity, i.e. one that can vary in time: the speaker's estimate of the pragmatic information of the addressee.

In the following pages, we will submit Dik's (1989) proposals for the assignment of pragmatic functions (mainly to be found in Ch. 13, pp. 263-287, but also in chapters on constituent ordering and prosodic features) to a careful examination with respect to (a) the theoretical question of how the interface between the static grammar and the dynamic theory of verbal interaction is handled with regard to pragmatic functions; (b) the practical question whether the reader finds a set of proposals that can be operationalized in the analysis of linguistic material. In considering these matters, we will limit ourselves to the two intra-clausal pragmatic functions and their subtypes, i.e. Topic and Focus.

The article will address three problems: firstly, the absence of a link between relevant aspects of the theory of verbal interaction and the pragmatic function assignment component of FG (\$1); secondly, the irreconcilability of the Given-New and the Topic-Focus distinctions (\$2); thirdly, the lack of any "special treatment" for Topics in English (\$3). Having pointed out the implications of these problems and drawn some novel conclusions, we continue $(\$ 4)$ by applying the proposals for pragmatic function assignment found in Dik (1989) to The story of Babar; the application is successful, but provides further evidence for the conclusions drawn in the preceding section.

\section{The interface between FG and the theory of verbal interaction}

The key notion common both to a grammar incorporating pragmatic function assignment and to a theory of verbal interaction is "discourse". This term, however, is understood in two different ways, depending on which approach is taken. Firstly, a discourse (usually then a count noun) may be seen as the product of text-creating activity; alternatively, discourse (now usually a mass noun) may be seen as the ongoing text-creating process itself. 
Grammarians, with their interest in the outcome of real-time operations and processes, generally take the former view. So also Dik (1989: 266-267), who identifies stories, monologues, etc. as discourses. He cites the product of his own labours as an example, i.e. the book he has written, and its chapters, subsections, paragraphs and ultimately its individual clauses -- all these are discourses, hierarchically organized with respect to one another. Through the identification of the clause as a mini-discourse, Dik paints a picture of a pyramidical structure, with an uninterrupted rank scale from clause to book.

Each discourse so identified stands in a relation of "aboutness" to an entity or group of entities in some "mental world" (see also Dik 1989: 46). These entities are termed D-Topics, and have the property of topicality. Dik (1989: 266) further recognizes "topical elements" in discourses. These are not defined, but we take them to be the particular part or parts of each discourse that is/are referentially or denotationally linked to one or more D-Topics. Any of these topical elements may (or, importantly, may not) be singled out for special treatment with respect to form, order or prosodic properties. This singling-out is operationalized in FG by the assignment of one of the pragmatic functions Topic or Focus. These have corresponding effects on the operation of the expression rules. Note, as a difficulty, that topical elements are thus determined per discourse (which may be much longer than a clause), while pragmatic functions are assigned per clause.

Alongside this grammarian's approach to discourses as the outcome of verbal activity, Dik (1989: 267, from $\$ 13.3$, par. 2 onwards) also adopts a view of discourse as an ongoing text-creating process. Here he suggests that as each stretch of discourse unfolds, so a "topic store", in some unspecified abstract sense, is "gradually ... filled with D-Topics as these are introduced". We take it that the unmentioned agent of the two passives in the quotation is the speaker/writer, at work in real whom?

${ }^{1} \mathrm{~A}$ question we will return to is: singled out by

${ }^{2}$ Here, we assume, discourse is taken to correspond to stretches considerably longer than one clause. 
time. As Dik's presentation continues, it becomes increasingly manifest that he does indeed intend the reader to imagine discourse production in real time: "... at some point ... for the first time ... then ... go on to talk about..." (1989, \$13.3, par. 3).

Thus, in one and the same section 13.3 , Dik both claims that D-Topics are phenomena that can be recognized retrospectively by the grammarian and assumes that the speaker/writer has D-Topics at her disposal, ready to be marshalled in ongoing discourse. Here, then, the reader finds himself at the cutting edge, right up against the interface between FG and the ambient theory of verbal interaction. The notion of D-Topic is, to judge by Dik (1989: 267, n. 5), inspired above all by Brown and Yule (1983: 71). These writers, however, differ from Dik in taking an exclusively process view on discourse (always used as a mass noun in their book). Indeed, they insist that the notion of a speaker having D-Topics at her disposal offers an unrealistic outlook on discourse production. Rather, they are interested in "the general pretheoretical notion of "topic"" as "what is being talked about" in a conversation. They further find it unlikely that their notion of a discourse topic will be identifiable with one part of a sentence. Brown and Yule's discourse topic is thus in essential respects different from Dik's entity-based notion with its implied correspondence with sentential constituents. ${ }^{3}$

The question now arises whether the real-time approach to pragmatic function assignment taken by Dik on p. 267 is conceptually compatible with most of the rest of $F G$, which takes the grammatically respectable retrospective view of language structure. As a model, FG is of course characterized by a "quasi-productive mode", being biased towards production rather than comprehension, but not being designed "necessarily [to] simulate the various steps that a speaker takes in producing linguistic expressions" (1989: 52). In Dik's account of the assignment of pragmatic functions, however, he appears no

${ }^{3}$ It is not unlikely that the very examples Dik and Brown \& Yule choose lead to different conclusions: Dik considers his own book, a highly structured and thoroughly planned written discourse, while Brown \& Yule concentrate on informal conversations and hastily composed memoranda. 
longer to observe a quasi-productive mode but rather to provide the elements of a theory of ongoing production. To our view, this disturbs the conceptual unity of the model, a point to which we will return in $\$ 2$, on the incompatibility of the pairs Given-New and Topic-Focus.

The central issue, from our perspective, concerns the relation between the range of available D-Topics and the instances of Topic assignment in the corresponding text. Given that topical elements are determined per discourse (in the clause-to-book sense adumbrated above), while pragmatic functions are assigned per clause, how is it determined which of the available topical elements is chosen as Topic? Let us consider whether either of the two perspectives taken, the retrospective view of the grammarian or the discourse analyst's interest in ongoing processes, offers an answer.

Looking retrospectively at discourses as products rather than processes, and specifically at individual sentences, Dik (1989: $268 \mathrm{ff}$. ) provides a set of criteria with which to identify a Topic, and further to subclassify Topics into four classes. By specifying various kinds of special treatment Topics may receive, he offers the practising linguist guidance in determining whether to assign Topic or not. As for the singling-out of topical elements referred to above, we understand Dik's position to be that it is not the speaker/writer who singles out elements for pragmatic function assignment, but the analyst. It is he who allocates Topic, Focus, etc. to constituents in representations. But his criteria for so doing remain obscure.

The other tack taken by Dik, which follows the realtime production of utterances in discourse, appears to offer above all a classification of D-Topics (i.e. entities) in terms of the result of their being mentioned. Not everything is fully clear (sec. 13.3, \$3): the first presentation of a D-Topic is termed a NewTop; an entity so introduced is a GivTop (Given Topic); similarly, an entity that is mentioned, temporarily neglected and later revived is termed a ResTop (Resumed Topic). Let us conclude, however, that what is intended is a classification of the entities held in the store of

${ }^{4}$ New Topic; for details of this function, and all the others introduced in $\mathrm{Dik}$ (1989: Ch. 13) see section 2 below. 
D-Topics in terms of their history of use in
corresponding discourse.

What neither perspective offers the reader is understanding of the speaker/writer's motivation in choosing one term rather than another as Topic of a particular utterance in discourse. We believe that this is the missing and vital link between the two perspectives adopted, between the two views of language as product and language as process. On the one hand, the grammarian's backward-looking view takes us only as far back in time as the form that results from production. On the other, the discourse analyst's forward-looking view fails to make the jump from the arrangement of D-Topics to the actual choice of Topic. The only connection that is achieved is feedback from choice of Topic to the classification of the store of D-Topics. In outline, we see the interface as follows:

Figure 1

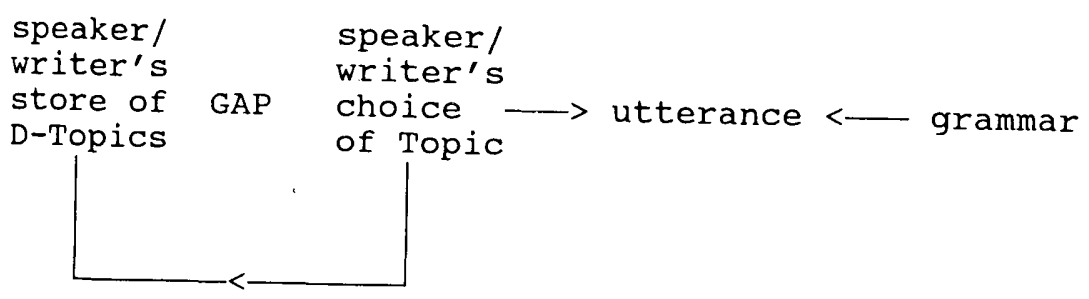

The purpose of this section has been to draw attention to the two lines of approach taken by Dik (1989) to discourse and to the assignment of pragmatic functions. our finding is that the two lines do not meet, that a gap arises between the set of D-Topics, classified in virtue of their use, and the actual choice of topic made by the speaker/writer per clause.

We note, however, that an attempt to bridge the gap has been made in a recent contribution to FG by Hannay (1990). Hannay adopts a clearly process-oriented approach, distinguishing a number of strategies available to the speaker for conveying information. In his view, there are five modes of message management, each of which induces distinct assignments of Topic and Focus. Such work aims to supply the missing link: it suggests how the speaker's choice of Topic may be constrained, while 
offering the analyst clear criteria for recognizing the effects of such a choice. This approach, based as it is on strategies, makes selection of Topic dependent upon the speaker's communicative goals. We should not forget, however, that not only such goals but also languagespecific constraints may influence Topic-selection strategies, as in Hungarian, where, according to Dik (1989: 363), following De Groot (1981: 45), P1 can harbour one or more Topic constituents. ${ }^{5}$ Thus, the speaker's assignment of Topic - and indeed of pragmatic functions generally - will always be a matter of balancing her needs with the specific requirements of the language in which she expresses herself.

\section{Topic and Focus function and the Given-New distinction}

\subsection{Introduction}

After this general assessment of the approach taken by Dik (1989) to discourse and to pragmatic function assignment, let us turn our attention to his proposals concerning the different types of pragmatic functions. Dik (1989: 265ff) combines in his clause-internal pragmatic functions information concerning the TopichoodFocushood of an entity and its Given-New status. Thus instead of the one-dimensional pragmatic functions Topic and Focus (cf. Dik 1978), he offers us the following range of hybrid pragmatic functions: Given Topic, SubTopic, New Topic, and Resumed Topic; New Focus and Contrastive Focus.

The exact relation between the Topic-Focus and GivenNew distinctions remains, however, unspecified. What is certain is that the pragmatic function Topic will be assigned to topical elements that are singled out for special treatment, the pragmatic function Focus to focal elements singled out for special treatment, and that there is a "partial correspondence" between the

${ }^{5} \mathrm{An}$ interesting research question would be to determine, for cases in Hungarian where more than one Topic is chosen, which corresponding term would be chosen for Topic assignment in English, given the same communicative setting and goals. 
dimensions of topicality and focality on the one hand and the Given-New distinction on the other (Dik 1989: 266). The correspondence presumably results from the fact that both the topicality-focality and the Given-New distinctions are definitionally related (either explicitly or implicitly) to the notion pragmatic information, and is reflected in the fact that topical elements tend to be given, while focal elements are typically new. The correspondence is, however, by no means complete: as will be clear from the list of hybrid pragmatic functions, topical elements may in fact be new, and focal elements may be given. And since Topic and Focus function are only assigned to certain topical and focal elements -- those singled out for special treatment -- the correspondence between Topichood-Focushood and Givenness-Newness may be expected to be even more "partial".

In the rest of this section, we will first of all give a summary of the various subtypes of Topic and Focus proposed by Dik, and of the special treatment these are given across languages. Secondly, we will discuss in some detail the differences between the pragmatic functions Topic and Focus and the Given-New distinction. Finally we will draw attention to a number of problems the practising linguist may encounter in the application of the pragmatic functions Topic and Focus in English, problems arising from the combination of two essentially different pragmatic dimensions into one set of complex pragmatic functions.

\subsection{Types of Topic and Focus}

The pragmatic function Topic is divided into four subtypes. First of all, there are New Topics (NewTops). These are described as "the first presentation of a DTopic" (Dik 1989: 267); they introduce a topical entity into the discourse. As such, NewTops are at one and the same time topical (in that they introduce a topical entity into the discourse) and focal (in that they introduce this entity; Dik 1989: 269). Their formal properties include a strong preference for taking late (or at least non-initial) position in the clause, and, in the spoken language, prosodic prominence. Thus, in chapter 18 (Dik, 1989: 391) we read that "the NewTop constituent captures the most prominent accent of the 
expression". The following sentences illustrate the kind of construction typically used to introduce a NewTop:

(1) I'm going to tell you a story about an elephant called Jumbo (NewTop) (Dik 1989: 268)

(2) Suddenly, right before our eyes, there appeared a huge elephant (NewTop) (ibid.)

Once an entity has been introduced into the discourse by means of a NewTop, it can be treated as a Given Topic (GivTop) in the subsequent discourse, ${ }^{6}$ where it must be "kept alive" through repeated reference. The grammatical means available for maintaining a GivTop include anaphoric reference, syntactic parallelism, switch reference and obviation (Dik 1989: 271-275). As far as their prosodic features in the spoken language are concerned, GivTops are not treated in any special way: they have no accentual prominence (unless contrasted to some other GivTop; see Dik 1989: 391). Finally, GivTops can be given special treatment with respect to order (position in the clause). In chapter 13 no mention is made of a special position for GivTops, but in chapter 16 (on constituent order) we find the following "specific principle" (Dik, 1989: 348f):

SP4: There is a universally relevant clause-initial position $P 1$, used for special purposes, including the placement of constituents with Topic or Focus function.

The general pattern for the uses made of $\mathrm{P} 1$ is described as follows:

${ }^{6}$ There appear to be cases where Given Topics are not (directly) introduced by New Topics, but by Sub-Topics. If the sub-Topic in question is introduced by (or rather inferrable from) a New Topic, one might say that the Given Topic is indirectly introduced by that New Topic. However, as we will see later (section 2.3), Sub-Topics need not be inferrable from New Topics. In that case the Given Topic is properly introduced by the Sub-Topic. 
(i) Languages often have designated categories of constituents which must be placed in P1 [In English: Q-words, subordinators, relative pronouns]

(ii) If $\mathrm{P} 1$ is not occupied by some P1-constituent, it may be used for constituents with (Given)Topic ${ }^{7}$ or Focus function.

The following text provides an example of a Topic chain (with GivTops, in the form of anaphors, appearing in P1)

(3) Yesterday I got a phone call from the tax inspector (NewTop). He/The man/The joker (GivTop) wanted me to come to his office, and he/p (GivTop) gave me the impression that I was in for some trouble (Dik 1989: 271)

The third type of Topic is the sub-Topic (SubTop). SubTop function is assigned to those entities that behave as if they had been explicitly introduced into the discourse (i.e. they behave like GivTops), but which instead are inferred from a GivTop (or NewTop) on the basis of our knowledge of what is normally the case in the world (Dik 1989: 267, 275). Since they behave as if they had been introduced into the discourse, we may assume that subTops receive the same special treatment as GivTops, except that in the spoken language they will "typically" have some degree of accentual prominence (on account of their having something "new" and "contrastive", Dik 1989: 391). Sentences (4) and (5) provide some typical examples of subTops.

(4) John gave a party (NewTop) last week, but the music (SubTop) was awful. (Dik 1989: 275)

(5) Mary got some picnic supplies (NewTop) out of the car. The beer (SubTop) was warm. (Dik 1989: 276)

${ }^{7}$ Not only Given, but also Sub- and Resumed Topics can be found in P1. The restriction, therefore, is surely not that the element must have Given Topic function, but that it must not have New Topic function (since these are typically placed in non-initial position). 
The final subtype is that of Resumed Topics. ResTops are GivTops that have not been mentioned for some time in the discourse. Thus, in addition to their own typical properties (strong anaphoric reference, indication that the entity has been mentioned before, and, like subTops, "typically" a degree of accentual prominence in the spoken language), they will receive the same special treatment as GivTops (Dik 1989: 277). Sentence provides a typical example:

(6) John had a brother Peter (NewTop) and a sister Mary (NewTop). Peter... [considerable episode about Peter]. Now, John's sister Mary, who I mentioned before... (ResTop) (Dik 1989: 277)

The pragmatic function Focus has also been divided into several types which are potentially relevant for the analysis of natural languages. First of all, a distinction is made between New (or Completive) Focus (NewFOC) and Contrastive Focus (ContrFoc). NewFoc is what we find in question-answer pairs such as the following:

(7) $\mathrm{X}$ : Where is John going?

$\mathrm{Y}$ : (a) John is going to the market (NewFoc)

(b) To the market (NewFoc) (Dik 1989: 279)

NewFoc is thus assigned to those pieces of information that are assumed to be completely new to the addressee. However, Focus information need not be entirely new; it may also include information already assumed to be available to the addressee, but focused on by virtue of some implicit or explicit contrast. In that case we speak of ContrFoc, of which there are two types. The first of these is Parallel Focus (ParFoc; example (8)); the second type is counter-presuppositional Focus, which in turn is subdivided into Replacing Focus (RepFoc), Expanding Focus (ExpFoc), Restricting Focus (RestrFoc) and selecting Focus (SelFoc) (examples (9)-(12)). 
(8) John and Bill came to see me. John (ParFoc) was

nice, but Bill (ParFoc) was boring. (Dik 1989: 278)

(9) X: John bought coffee.

Y: No, he bought rice. (RepFoc) (Dik 1989: 283)

(10) $X:$ John bought coffee.

Y: Yes, but he also bought rice. (ExpFoc) (Dik 1989: 284)

(11) X: John bought coffee and rice.

$Y$ : No, he only bought coffee. (RestrFoc) (ibid.)

(12) X: Would you like coffee or tea?

Y: Coffee (SelFoc), please. (ibid.)

With regard to special treatment, Focus function may manifest itself (cross-linguistically speaking) through one or more of the following focalizing devices (Dik 1989: 278):

(i) prosodic prominence: emphatic accent [in the spoken

(ii) special constituent order

(iii) special Focus markers

(iv) special Focus constructions (to be discussed in Dik (forthcoming))

The first of these, prosodic prominence, is the most common focalizing device. In English it applies to both New (Completive) Focus and to all types of contrFoc (Parallel, Replacing, Expanding, Restricting, Selecting). In addition, Focus constituents may appear in P1; unlike Topic constituents, they must have prosodic prominence.

\subsection{Differences between Topic-Focus and Given-New}

We already noted that the correspondence between the pragmatic functions Topic and Focus and the dimensions Given and New is only partial. In what follows we will maintain that the two pairs of notions correspond only in that they are both pragmatic in nature, i.e. they concern the informational status of discourse elements in relation to the "wider communicative setting" in which they are used -- which in turn can be understood in terms of the addressee's pragmatic information at the moment of speaking (Dik 1989: 265). Apart from being pragmatic in nature, the two pairs of notions are, as we will show, essentially different; even to the extent that it is 
doubtful whether they should (or can) be combined into one set of complex pragmatic functions.

Let us first of all look at the way these notions are defined. In both cases reference is made (explicitly or implicitly) to the term pragmatic information, i.e. to "the full body of knowledge, beliefs, assumptions, opinions and feelings available to an individual at any point in the interaction" (Dik 1989: 9). The notions Given and New are defined in terms of what is assumed to be part of or inferrable from a person's pragmatic information (Dik 1989: 265-266). The pragmatic functions Topic and Focus, on the other hand, are defined in terms of "special treatment": they are assigned to those topical and focal elements "singled out for special treatment with regard to form, order and prosodic properties" (Dik 1989: 266). Topical elements are those elements "labout" which information is given in the discourse"; focal elements are "those pieces of information which are the most important or salient with respect to the modifications which the speaker wants to effect in the pragmatic information of the addressee" (ibid.).

Thus, unlike the notions Given and New, Topic and Focus assignment involve a selection from the available topical and focal elements. This immediately leads to a difference between the two notions with regard to the problem noted in $\$ 1$. While in the case of Topic and Focus assignment the failure to fill the gap between the available topical and focal elements and the assignment of Topic and Focus function made it impossible for the two views of discourse -- as the product of text-creating activity and as the ongoing text-process itself -- to connect, this problem does not arise in the case of the Given-New distinction. For although given and new elements are typically expressed in such a way as to reflect their (assumed) Given-New status (in accordance with Clark and Haviland's (1977) "Given-New Contract"), assigning Given or New status to an element does not involve any "singling out" of elements for special treatment. As a result there is no "missing link": the two views of discourse are perfectly reconcilable. The outcome of the text-creating process, which the grammar seeks to describe and explain, may be seen as (superficially) reflecting the text-creating process itself, and "psychological adequacy" is attained.

Another complicating factor is that the Given-New 
distinction is not a simple, unitary phenomenon; to distinguish merely between Given and New is to disregard the complexity of these notions. This complexity is first of all reflected in the fact that the distinction between Given and New can be made at various levels. Thus, Given has been defined (in the narrowest sense) in terms of that which can be assumed to be in the consciousness of the addressee at the time of speaking (Chafe 1976). In a somewhat wider sense it includes that which is "recoverable from the discourse" (Halliday and Hasan 1976, Halliday 1985), that which is "activated in the discourse" (Brown and Yule 1983) or that which is "discourse-bound" (Hannay 1985a). In its broadest sense, givenness is defined in terms of what is assumed to be part of knowledge shared by speaker and addressee (Clark and Haviland 1977, Prince 1981). Dik clearly opts for the broadest interpretation: he defines the distinction in terms of the speaker's estimate of the addressee's pragmatic information. This means that Given and New must be understood not merely in terms of what is in the addressee's consciousness, or even in the discourse, but in the addressee's "full body of knowledge".

This "full body of knowledge", a person's pragmatic information, consists of three components (Dik 1989: 9):

- general information: long-term information concerning the world, its natural and cultural features, and other possible or imaginary worlds

- situational information: information derived from what the participants perceive or otherwise experience in the situation in which the interaction takes place

- contextual information: information derived from the linguistic expressions which are exchanged before or after any given point in the verbal interaction

From this it follows that, like pragmatic information, given information can also be divided into three types. Thus, we can distinguish between discourse entities that are "Contextually Given" (as in example (13)), "Situationally Given" (example (14)), and "Generally Given" (example (15)):

(13) I saw Mary yesterday. She told me she had bought a new car.

(14) Could you pass me the salt please.

(15) The Prime Minister has just resigned. 
The fact that in examples (14) and (15) the elements the salt and the Prime Minister are at the same time given (situationally and generally, respectively) and new (contextually) shows that it is not enough to distinguish merely between Given and New.

Another reason for distinguishing different types of givenness is that there seems to be a difference in "degree of givenness" between the various types. Thus, according to Prince's (1981) Familiarity scale, generally given entities (Prince's Unused entities) have a lower degree of givenness than contextually and situationally given entities. Moreover, according to what cruse (1980) calls the "order of precedence" among the various types, situationally given entities are in turn "less given" than contextually given entities. Thus, whenever a term has two possible referents, one introduced contextually, the other situationally, the intended (and chosen) referent will normally be the one introduced by means of the context. Similarly, when the referent of a term can be retrieved from the situation or from the addressee's general knowledge, it will normally be retrieved from the situation. This difference in degree of givenness is reflected in the way the different types of entities can be pronominalized: contextually given entities can be referred to by means of both anaphoric and deictic pronouns, situationally given entities only by means of deictic pronouns, while reference to a generally given entity always requires the use of a full description. Moreover, it appears that in certain languages (e.g. the Scandinavian languages) as well as in a number of dialects (the Frisian dialect Fering, the German dialect Amern) there are two different forms of the definite article, one of which is used with textually or situationally given entities, the other with generally given or inferrable entities (Ebert 1970).

In other words, there is sufficient evidence to suggest that a distinction between different types of given and new is necessary. However, no such distinction is made in the two-dimensional pragmatic functions proposed by Dik: Topics are either given, inferrable or new; Focus elements are either new or given (contrastive). To complicate matters even further, topicality (a prerequisite for Topichood) is defined as characterizing those entities about which information is provided in the discourse; focality (a prerequisite for Focushood) as attaching to those pieces of information that are the 
most salient with regard to the addressee's pragmatic information. In other words, candidates for Topic function must be drawn from the discourse (textual and, possibly, situational information); Focus function, on the other hand, is assigned to the most salient information in the clause, irrespective of whether it concerns textual, situational or general information. In the next section $(\$ 2.3)$ some of the consequences of these discrepancies will be illustrated.

A final difference between Topichood on the one hand and Given-New status (and Focushood) on the other concerns the fact that whereas Given-New status (and Focus function) can be assigned to terms and predicates (and combinations of the two) alike, Topic function can only be assigned to expressions referring to discourse entities (including terms). This difference, which we take to have been inherent in FG since its inception, follows from the claim that topicality characterizes entities while focality attaches to pieces of information (Dik 1989: 266). As a result, GivTops can only be introduced by other entities (NewTops), and SubTops only inferred from other entities (NewTops or GivTops). However, a person's pragmatic information consists of more than a list of discourse entities. It includes a person's full body of knowledge, beliefs, feelings etc. at a particular moment; i.e. not only entities, but also properties of those entities, relations between different entities, SoAs in which those entities play a role, etc. This knowledge will in part be specific knowledge of particular entities and events; but in part it will organized in general knowledge structures relating to particular kinds of entities and events, constructed on the basis of prior experiences. In addition, our pragmatic information contains plans and goals, motivations and strategies, etc. Comprehension of a discourse involves activation of and drawing inferences from large parts of this knowledge. The following example illustrates both the number and the different kinds of inferences that need to be drawn to comprehend even the simplest messages:

(16) (Mary and John are having breakfast together. Mary says to John:)

Pass me the cereal, please. (Löbner, 1985: 319)

The definite term the cereal may in fact have a number of 
possible referents: both Mary and John may have cereal on their plates, there will be cereal in the pack (or packs) on the table, and there may even be a spare pack on a shelf nearby. Nevertheless John may be expected to know which cereal to pass on account of his knowledge of what people (in general, or Mary and he in particular) usually do in similar situations, his knowledge of Mary's goals and motivations, and his knowledge of her preference for a particular kind of cereal. Likewise, in the following example, there may be more than one window, only one of which is open. The reference is, therefore, unambiguous, but only on account of the use of the predicate close in combination with the knowledge that only open windows can be closed.

(17) Could you close the window, please?

It will be clear that understanding discourse depends for a large part on activation of and drawing inferences from whatever pragmatic information we have available. This inevitably means that many pieces of information may be assumed to be given or inferrable on account of this information (and will be treated as such) which do not qualify for Topichood, either because the particular given or inferrable piece of information is not referred to by means of a term (i.e. is not an entity), or because the information referred to has not been introduced by means of another term (i.e. by means of a NewTop). The consequences of this strict approach to Topic assignment (in particular with regard to subTops) will be discussed below.

\subsection{The relation between Topic-Focus and Given-New: some problems}

We will now take the point of view of the analyst, and try to apply some of the subtypes of Topic and Focus described above to English data. We will pay particular attention to problems and/or inconsistencies arising from the combination of information concerning the Topic-Focus function and the Given-New status of discourse elements in one pragmatic function. 
Given Topics

GivTops, as we have seen, are defined in terms of special treatment of certain topical elements, whereas givenness is defined in terms of the speaker's estimate of the addressee's pragmatic knowledge. Nevertheless, the givenness of GivTops turns out to be restricted to "contextually given" information, i.e. information that has been introduced into the discourse by means of a NewTop. One might consider, however, that entities introduced by the situation, or present in the general pragmatic information of the addressee are also GivTops: they are given (in terms of pragmatic information), and they (formally) behave like Topics (they may have P1 position without having prosodic prominence). Thus in the following examples

(18) Watch out! The ceiling is caving in!

(19) (Guide in a museum): This painting was painted by Turner in 1844. It is called 'The Great Western Railway'. The painting has been praised for...

(20) Have you heard the latest news? The Berlin Wall has been demolished.

the entities referred to by means of the italicized terms are contextually new. In the theory of Dik, they can therefore only be assigned NewTop or NewFoc function. However, they do not have emphatic accent or the most prominent accent of the expression (though, like subTops and ResTops they may "typically" have a certain degree of accentual prominence, on account of the fact that they are contextually new). They do go into P1. Moreover, we can certainly think of these elements as topical elements, as what the discourse is about (see in particular example (19)). In other words, it seems justified to assign these constituents GivTop function. The only difference between these elements and Dik's GivTops is that the entities they refer to have not been introduced by means of the discourse (through a NewTop), but have either been introduced by means of the situation, or are assumed to be available on account of the fact that they are part of the addressee's general information. If so, three kinds of GivTop could be distinguished: Contextually GivTops, Situationally GivTops and Generally GivTops. In that case, GivTop function would be more directly related to the addressee's (contextual, situational and general) 
pragmatic knowledge.

Sub-Topics

SubTops are defined by Dik as "Topics which may be legitimately inferred from a GivTop on the basis of our knowledge of what is normally the case in the world" (Dik 1989: 275), and are called in full "SubTops of the GivTop". Inferrability, however, is related to givenness rather than to Topichood (cf. Prince 1981). Thus an element may be inferrable (in the sense that it can be inferred from the addressee's entire pragmatic information, including information provided in the discourse), without necessarily being inferrable from a Giv'Top. There is no reason, however, to assume that such elements are not SubTops, since they do qualify for this function on account of their formal properties. Thus, in the following examples, the italicized terms behave like SubTops. They are not, however, inferrable from other discourse entities (GivTops or NewTops), but from predicates (which, as we have seen in $\$ 2.2$, cannot be Topics) or certain combinations of predicates and arguments:

(21) It was dark and stormy the night the millionaire was murdered. The killer left no clues for the police to trace (Carpenter and Just 1977; see Brown and Yule 1983: 258)

(22) Mary dressed the baby. The clothes were made of pink wool (Sanford and Garrod 1981)

(23) We wanted to buy the house straightaway, but the estate agent advised us to wait a bit.

What we suggest, therefore, is that subTops should be defined as "inferred Topics" rather than as "SubTops of the GivTop". Note that in either case subTops are required to behave as if they were (Given) Topics: they must have P1-position (with at most a "certain degree" of accentual prominence). This means that, contrary to what is suggested by Dik in footnotes 6 and 12 (Dik 1989: 267, 275), not all inferrables are subTops. Indeed, it appears that many inferrables are (according to the special treatment requirement) neither (Sub-/Given) Topic nor Focus (see example (24)); they may, however, have NewTop function (i.e. they introduce an entity into the discourse; see example (25), and indeed (26)). 
(24) There was a car approaching the junction, but the driver didn't stop at the give way sign (Brown and Yule, 1983: 183)

(25) A: What did you see in the circus?

B: Well, there was an elephant that amazed us with his tricks. His name was Jumbo. He could stand on his trunk...

New Topics

Here, too, New is new at a contextual level. Thus NewTops may at the same time be given or inferrable with regard to the addressee's general or situational pragmatic information, as illustrated by the following examples respectively:

(26) Yesterday in the pub I met your sister Mary (Dik, 1989: 269)

(27) Look at that man over there! He's talking to himself.

Focus

We have seen that Focus is subdivided into NewFoc and ContrFoc. Once again the term New is rather misleading, as what is meant is not new with regard to the addressee's entire pragmatic information, but new in a textual sense. ContrFoc is, by implication, contextually given (see Dik 1989: 282, schema (43)): the information focused on is not completely (i.e. contextually) new to the addressee; it is focused on by virtue of some implicit or explicit contrast.

This distinction turns out to be somewhat problematic. In the first place, it appears that contrFoc constituents need not be contextually given, but may be situationally given (and contextually new, as in example (28)), generally given (and contextually new, as in (29)), or completely new to addressee (as in (30)) :

(28) (guide in a museum:) This (painting) is almost certainly an authentic Rembrandt. This one has probably been painted by one of his pupils.

(29) Have you heard the latest news? George Bush is going to resign; Dan Quayle is going to succeed him. 
(30) A: What are you going to buy?

B: I'm going to buy a book for peter, a record for Sally, some flowers for my mother...

Secondly, Given Focus elements are not necessarily used contrastively: Thus, in the following example, the given element her is clearly the most salient information in the clause. Moreover, it is singled out for special treatment: it has emphatic accent (in spoken language), and it appears in what may be considered a special Focus construction. It is not, however, used contrastively:

(31) A: What about Rebecca?

B: It was to her that John gave his most precious painting. (Hannay 1983: 214)

The problem thus seems to be that the correspondence between the Given-New distinction and the [ feature is, once again, only partial. As a result, there is no NewFoc-ContrFoc opposition (as suggested by schema (43), Dik 1989: 282). According to Hannay (1983: 210), the two distinctions (Given-New vs ContrastiveNoncontrastive) actually relate to two different levels. The first level (which Hannay calls "assertive Focus", cf. De Jong 1981) relates to elements that are new for the addressee in the given setting (cf. Dik's Completive Focus). The second level (which Hannay calls "Emphatic Focus") relates to information which is "important" or "salient" in that it is emphasized in the given setting. These two levels do not represent a dichotomy: both new and given information may be emphasized, whereby Emphatic Given Focus typically, but not necessarily, involves contrast. ${ }^{8}$ According to such a categorization of Focus

can be further subdivided according to contrast classification (i.e. into Parallel Focus and counterpresuppositional Focus). Notice that New (Completive) Focus can also be divided into several subtypes. First of all a distinction can be made between Non-Emphatic New Focus (Assertive Focus, as in example (7)) and Emphatic New Focus; secondly, Emphatic New Focus can be subdivided into Contrastive New Focus (Parallel Focus, see example (30)) and Non-contrastive New Focus, as in: 
types, sentence (31B) would be an example of an Emphatic Given Focus which does not involve contrast.

\section{Special Treatment}

The difficulties one may encounter in the assignment of Topic and Focus are, however, not only due to fundamental differences between the Topic-Focus and Given-New distinctions. In addition, there are problems arising from the requirement that Topic and Focus elements be "singled out for special treatment". Thus we run into problems in assigning pragmatic functions in a sentence like (32) $(=(8))$ :

(32) John and Bill came to see me. John was nice, but Bill was rather boring (Dik 1989: 278)

In Dik's theory, the contextually given elements John and Bill are to be assigned Parfoc: they are focal elements singled out for special treatment (prosodic prominence in spoken language, P1 position). They do, however, also qualify for Topic function (they are topical elements placed in $\mathrm{P} 1 ;$ the prosodic prominence is due to contrast). This overlap is possible because we are dealing with four different dimensions: givenness in terms of presence in preceding context, focality in terms of salience, topicality in terms of "what the discourse is about" and Topic-Focus in terms of special treatment. The constituents John and Bill in the second sentence of example (32) have all these features. Thus it appears that there is not only an overlap between topicality and focality, but also between Topic and Focus. Whether the theory allows us to assign John and Bill in (32) both Topic and Focus function is not altogether clear. In chapter 13 Dik is rather vague on this point. All he says is that "the constituents John and Bill are emphasized, although they have already been introduced and may thus be assumed to be Given Topics to A" (Dik 1989: 278; underlining is ours). In chapter 18, however, Dik seems to suggest that GivTop function can be assigned to contrasted elements: "a GivTop will have no accentual

(i) John bought a car (of all things). (Hannay 1983: 210) 
prominence (unless contrasted to some other GivTop)" (Dik 1989: 391; underlining is ours). If, however, constituents can at the same time have both Topic and Focus function, does this mean they should have the special (formal) features of both? And what, in that case, is the use of assigning pragmatic functions? If, on the other hand, constituents can have only one pragmatic function, how does one select the right pragmatic function in a sentence like (32)?

One way to solve this problem would be to redefine Topic and Focus in such a way that they become more consistently applicable. The first step would be to recognize that since special treatment of Topic and Focus may coincide (as in example (32)), both functions must be given a definition that is not solely dependent on special treatment of certain topical or focal elements. obviously, such a definition would require that the relation between topicality-focality and Topic-Focus be specified. In other words, what is needed is a specification of the speaker's strategies and motivations in singling out certain topical or focal elements for Topic or Focus assignment (possibly in terms of "relevance" or "aboutness", see Hannay 1990). If feasible, such a redefining of the pragmatic functions Topic and Focus would have the additional advantage of providing the "missing link" referred to in $\$ 1$.

We are, however, by no means certain that such a definition of Topic and Focus is actually feasible. Therefore we want to offer an alternative solution, which, in our view, will be consistent both with the theory of FG and with the data presented in the preceding sections. The solution we have in mind consists in abandoning the idea of Topic function in English. This may appear to be a radical proposal, but let us consider the evidence. We know that Topic and Focus are defined in terms of special treatment. We also know the forms this special treatment may take across languages (\$2.1). Let us now confine ourselves to English. With respect to Focus there does not seem to be any difficulty: although English does not have any special Focus markers, the remaining focalizing devices (prosodic prominence in spoken language, constituent order, special Focus constructions) provide sufficient means by which to single out certain focal elements. With Topics, however, the situation is different. First of all, GivTops do not have prosodic prominence (unless contrasted to some other 
Topic, but then prosodic prominence does not distinguish them from Focus elements). SubTops and ResTops may have some degree of accentual prominence, but this prominence is only "typical", and applies only to spoken language. Secondly, of the grammatical means that languages provide for maintaining Topic continuity mentioned by Dik (anaphoric reference, syntactic parallelism, switch reference and obviation), only anaphoric reference seems to apply to English. Note, however, that anaphoricity as such need not indicate Topichood: anaphors can be used to refer to contextually given (or inferrable) entities; these need not be Topic. We may therefore conclude that special treatment for Topic constituents in English consists in placement in P1 (but without prosodic prominence); a conclusion supported by the fact that in the examples provided by Dik the GivTop constituents are indeed always ${ }^{9}$ placed in this position. If this is the case, then Topic assignment is necessarily a rather arbitrary affair. After all, PI position can be filled only once, and thus Topic assignment can only take place in those cases where P1 is not already filled by some other element (either an element that would obligatorily go into P1, such as relative pronouns or subordinators, or, possibly, a Focus element). Obviously, this is not a desirable situation.

One may, of course, object that it is nowhere explicitly stated that Topic elements must go into P1; this is, however, something that must be deduced from what is stated in Dik (1989) about P1 position and from the fact that Topic assignment necessarily involves singling out elements for special treatment. Note, however, that dropping the requirement that Topics must be placed in P1 would mean losing the last possibility for giving special treatment to Topic elements in English.

The next question to ask might be whether our proposal to reject Topic assignment in English also covers NewTops. The answer is "yes", the reason being that it would be far more consistent, even with Dik's own proposal, to regard NewTop function as a particular kind of Focus. Notice, for instance, that if Topic assignment necessarily involves special treatment, NewTops are, strictly speaking, not Topics. They differ from GivTops,

${ }^{9}$ With one exception on p. 217 (ex. 18B). 
SubTops, and ResTops in that their Topic status does not depend on special treatment (non-initial treatment is "typical, preferred"; prosodic prominence, relevant only in spoken language, does not distinguish them from Focus constituents), but on whether or not a topical entity is being introduced into the discourse. However, as Dik himself observes, NewTop entities are in this respect both topical and focal; after all, focality is defined as "attaching to those pieces of information which are the most important or salient with respect to the modifications which $S$ wishes to effect in $\mathrm{P}_{\mathrm{A}}$, and with respect to the further development of the discourse (Dik 1989: 266, underlining is ours). And since their formal features do not distinguish them from Focus elements, there does not seem to be any reason not to regard them as a particular kind of (New) Focus (e.g. Presentative Focus $\left.^{10}\right)$.

Finally, let us consider the consequences of our proposal to reject Topic assignment in English for the theory of FG. First of all one may wonder whether it is not inconsistent with the theory to accept that some languages have two clause-internal pragmatic functions, whereas other languages (such as English) have only one. We claim that it is not. In the first place, it seems to add to the descriptive as well as to the typological adequacy of the theory to allow pragmatic function

${ }^{10}$ Notice that New Topic is simply another term for Hannay's "Presentative function", which is defined as follows

A term with presentative function refers to an entity which the speaker by means of the associated predication wishes to explicitly introduce into the world of discourse (Hannay 1985b: 171).

Dik uses the term New Topic "in order to stress its position in the strategies for introducing, maintaining and renewing "discourse topics"" (Dik 1989: 179). However, since Topic and Focus function are defined in terms of special treatment, and elements with Presentative function are treated as Focus rather than Topic elements, it will be more consistent to regard the pragmatic function Presentative as a subtype of New Focus (Presentative Focus). 
assignment to differ from language to language. Secondly, it brings pragmatic functions into line with, for instance, syntactic functions. After all, subject and object function are not relevant in all languages either. Thus, subject function is only considered relevant if the language in question has an active-passive opposition; and object function is only deemed relevant if the language allows for a Recipient object (Dik 1989: 219). Similarly, we may think of Topic and Focus function as relevant only if the language in question provides the means to single out certain topical or focal elements for special treatment. Since English provides only focalizing devices, it follows that only Focus function is

As far as the placement of elements in P1 is concerned, our proposal does not seem to have serious consequences either. According to Dik (1989: 349), "all languages may be supposed to use P1 for special purposes" (see also $\mathrm{SP} 4)$. In $\$ 2.1$ we already described the general pattern for the uses made of P1: it is either filled by some P1constituent, or by a constituent with Topic or Focus function. ${ }^{12}$ However, rejecting Topic assignment in

"Drawing the parallel with syntactic (and also semantic) functions even further, we may consider revising the pragmatic function hierarchy (Dik 1989: 34) in such a way that Focus is presented as more "central" than Topic (in analogy with subject and Agent in the syntactic and semantic function hierarchies, respectively). As we have looked at English data only, such a revision can, of course, only be hypothetical. The hypothesis is, however, an intuitively attractive one. After all, whenever clauses consist of one constituent only, this constituent, presenting the most salient information, will be assigned Focus function. Similarly, instance, in telegrams), it is focal information (from which Focus elements are selected) that is expressed rather than topical information (providing candidates for Topic function).

${ }^{12}$ Notice that, for English, SP4 is valid as it stands provided it is understood that Topic assignment is not necessarily relevant in all languages. The same holds, as we will see, for SP5. This principle, too, will still be 
English means that in many cases P1 will be occupied by a constituent which has no special pragmatic significance and which is not a P1-constituent. This raises the question of what "special use" is made of P1 in such cases, and of what determines placement in P1. The answer is provided in Dik's SP5:

SP5: Since the subject is the prime Given Topic candidate, it will often be placed in P1; this may lead to a reinterpretation of $\mathrm{P} 1$ as the unmarked subject position.

As far as English is concerned, we may therefore assume that in the unmarked case P1-position is filled by the Subject; and that in the marked case it will be filled either by a P1-constituent or by a constituent with Focus function. Thus we conclude that rejecting Topic assignment in English is not incompatible with the theory of FG in general, and pragmatic function assignment and P1-placement in particular.

\section{An application of Dik's (1989) proposals}

\subsection{Principles of analysis}

In the preceding section, we examined the internal consistency of Dik's (1989) proposals for the assignment of pragmatic functions, and suggested a number of ways in which those proposals might be adapted, specifically by disconnecting the Given-New and Topic-Focus parameters and by making it possible for the assignment of specific pragmatic functions not to apply in particular languages.

In this section, we will attempt to apply Dik's proposals, without any of the major adaptations suggested in $\$ 2$ and $\$ 3$, to a passage of running text with a view to testing their empirical value. The text chosen is the

valid, as long as one accepts that P1 can be interpreted as the unmarked subject position even for languages in which Topic function is not relevant. 
Story of Babar, ${ }^{13}$ which has the advantage of containing little syntactic complexity.

In the analysis, all P1 fillers have been indicated in heavy type. Our analysis is based on the following assumptions, which in turn are derived from a reading of Dik (1989):

1. For every finite clause, P1 must be filled (cf. Dik 1989: 362); this also applies to non-finite clauses containing designated categories, such as while washing the dishes, ....

2. P1 may contain no more than one constituent of the clause, ${ }^{14}$ and this constituent is always the first intra-clausal constituent.

3. Generally, the presence or absence of a comma after candidates for P1-placement has been taken as indicative of P2 (extra-clausal) and P1 (intraclausal) status respectively.

4. P2 differs from $P 1$ in accepting more than one constituent, as in the following example from the story of Babar:

$\{\text { (After dinner) Time, (because he is very tired) Reason }\}_{\mathrm{P} 2}$, he $_{\mathrm{P} 1}$ goes to bed. (11. 40-41)

5. Clause coordinators such as and, but, so and for are analysed as being inter-clausal, and as such not occupying any position in the clausal pattern.

${ }^{13}$ As presented in C. Fadiman (ed.), The world Treasury of Children's Literature, I , Boston \& Toronto: Little, Brown \& Co, 1984, pp. 133-141. The story has been translated from the French; we consider that the text of the translation is such natural English that the fact of its being a translation should not detract from the value of our findings. Furthermore, there are in Fadiman (1984) a number of illustrations to which, from time to time, the text makes allusion. Again, we do not consider that our failure to reproduce these illustrations affects the value of our findings.

${ }^{14}$ In clauses containing only one constituent, that constituent is, given assumption 1, consequently in P1. 
On the basis of these assumptions, our analysis has proceeded as follows:

1. Where P1 is occupied by a question word, subordinator, relative pronoun, satellite, dummy it or verbal constituent, $\mathrm{P} 1$ is indicated in heavy type; where the Pl-filler also carries a pragmatic function, there is an additional indication of that pragmatic function.

2. Constituents not in $P 1$ but bearing a pragmatic function [NewTop or NewFoc] are enclosed in square brackets, with an indication of the pragmatic function in question.

3. In keeping with our reading of Dik (1989), cf. $\$ 2.3$, we assign GivTop, SubTop and ResTop only to P1fillers.

4. Focus function is assigned only where there is evidence in the written text for special treatment of the constituent in question (e.g. P1-placement; parallelism; a preceding colon; etc.). Focus would of course be assigned much more frequently in a transcription of a reading of the story, since prosodic prominence is indicative of the presence of this function.

The analysis is followed by a commentary on each paragraph.

\subsection{The story of Babar analysed}

The story of Babar the little elephant

Jean de Brunhoff

translated from the French by Merle s. Haas

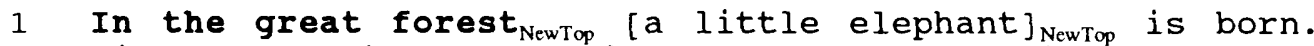

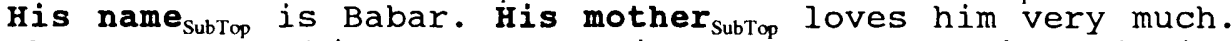
she $_{\text {GivTop }}$ rocks him to sleep with her trunk while singing softly to him.

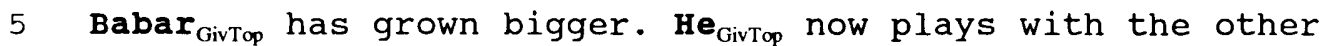

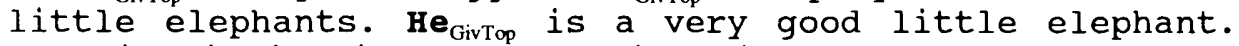
See him digging in the sand with his shell.

Babar $_{\text {GivTop }}$ is riding happily on his mother's back when [a 
wicked hunter, hidden behind some bushes] $]_{\text {NewTop' }}$ shoots at

10 them.

The hunter ${ }_{\text {GivTop }}$ has killed Babar's mother! The monkey ${ }_{\mathrm{ParFo}}$ hides, the birds ${ }_{\text {ParFoc }}$ fly away, Babar ParFoc $_{\text {cries. The }}$ hunter ${ }_{\text {GivTop }}$ rushes up to catch poor Babar.

Babar $_{\text {GivTop }}$ runs away because he is afraid of the hunter.

15 After several days, very tired indeed, he GivTop $_{\text {comes to a }}$ town.

He $_{\text {GivTop }}$ hardly knows what to make of it because this is the first time that he has seen so many houses.

so many things $s_{\text {New }}$ are new to him! The broad

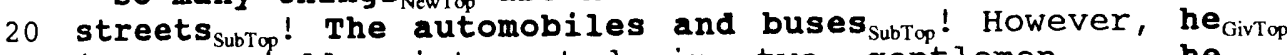
is especially interested in two gentlemen NewTop $_{\text {he }}$ GivTop notices on the street.

He $_{\text {GivTop }}$ says to himself: "Really, they GivTop $_{\text {are very well }}$ dressed. $I_{\text {GivTop }}$ would like to have [some fine clothes] NewTop'

25 too! $I_{\text {GivTop }}$ wonder how I can get them?"

Luckily, a very rich old Lady who has always been fond

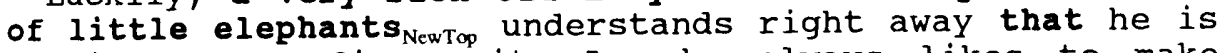
longing for a fine suit. As she always likes to make people happy, she $_{\text {GivTop }}$ gives him her purse. Babar GivTop $_{\text {says }}$

30 to her politely: "Thank you, Madam."

without wasting any time, Babar $_{\text {GivTop goes [into a big }}$ store $]_{\text {NewTop. }}$ He $_{\text {GivTop }}$ enters the elevator. It is such fun to ride up and down in this funny box, that he rides [al] the way up] ParFo $_{\text {[ten }}$ [imes] $]_{\text {ParFoc }}$ and [all the way down $]_{\text {ParFoc }}$

35 [ten times] ParFo. He $e_{\text {GivTop }}$ does not want to stop but the elevator boy SubTop finally says to him: "This GivTop $_{\text {is not a }}$

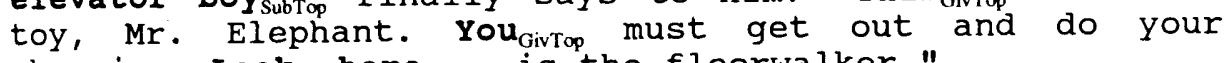

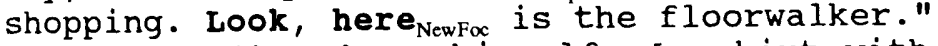

Babar $_{\text {GivTop }}$ then buys himself: [a shirt with a collar and

40 tie, a suit of a becoming shade of green, then a handsome derby hat, and also shoes with spats] $]_{\text {NewTop }}$.

well satisfied with his purchases and feeling very happy, Babar ${ }_{\text {GivTop }}$ now goes to have his picture taken.

And here $e_{\text {NewFoc }}$ is his photograph.

45 Babar $_{\text {GivTop }}$ dines with his friend the old Lady. she GivTop thinks he GivTop looks very smart in his new clothes. After dinner, because he is tired, he $\mathbf{e}_{\text {GivTop }}$ goes to bed and $\phi_{\text {GivTop }}$ falls asleep very quickly.

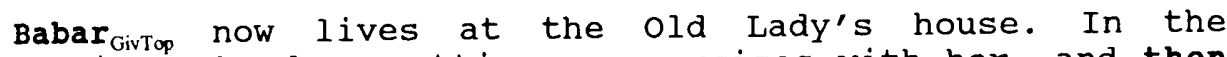
50 mornings, he does setting-up exercises with her, and then he takes a bath.

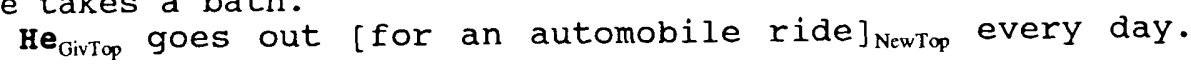


The old Lady GivTop $_{\text {has }}$ given him the car. She $\mathbf{G}_{\text {Givop }}$ gives him whatever he wants.

55 A learned professor ${ }_{\text {NewFoc }}$ gives him lessons. Babar $\mathbf{G}_{\text {GivTop }}$ pays attention and $\phi_{\text {GivTop }}$ does well in his work. $\mathbf{H e}_{\text {GivTop }}$ is a good pupil and $\phi_{\text {GivTop }}$ makes rapid progress.

In the evening, after dinner, he GivTop $_{\text {tells the old }}$ Lady's friends all about his life in the great forest.

60 However, Babar $_{\text {GivTop }}$ is not quite happy, for he $\mathbf{e}_{\text {GivTop }}$ misses playing [with his little cousins] NewTop $_{\text {and }}$ his friends, the

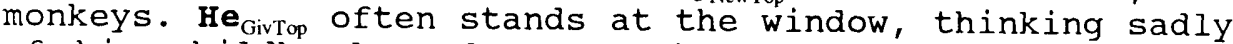
of his childhood, and $\phi_{\text {GivTop }}$ cries when he remembers his mother.

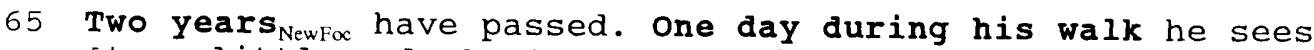
[two little elephants] NewTop $_{\text {coming toward him. They }}$ GivTop have no clothes on. "Why," he GivTop says in astonishment to the old Lady, "it's Arthur and celeste, my little cousins!"

70 Babar $_{\text {GivTop }} k i s s e s$ them affectionately and $\phi_{\text {GivTop }}$ hurries off with them to buy them [some fine clothes] ${ }_{\text {NewTop }}$.

He $_{\text {GivTop }}$ takes them to a pastry shop to eat some good cakes.

Meanwhile, in the forest, the elephants NewTop $_{\text {are calling }}$

75 and hunting high and low for Arthur and Celeste, and their mothers SubTop $_{\text {are }}$ worried.

Fortunately, in flying over the town, an old marabou bird $_{\text {NewTop }}$ has seen them and $\phi_{\text {GivTop }}$ comes back quickly to tell the news.

80 The mothers of Arthur and celeste $e_{\text {GivTop }}$ have come to the

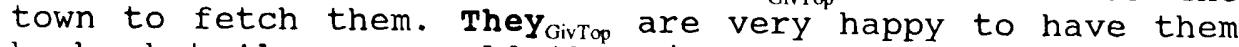

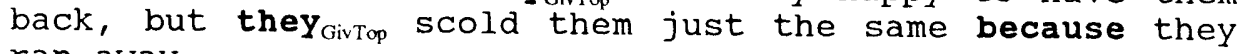
ran away.

Babar $_{\text {GivTop }}$ makes up his mind to go back with Arthur and 85 Celeste and their mothers to see the great forest again. The old Lady GivTop helps him to pack his trunk.

They $y_{\text {SubTop }}$ are all ready to start. Babar GivTop $_{k i s s e s}$ the old Lady good-bye. $\mathbf{H e}_{\text {GivTop }}$ would be quite happy to go if it were not for leaving her. He GivTop $_{\text {promises to come back }}$ 90 some day. $\mathbf{H e}_{\text {GivTop }}$ will never forget her.

They $_{\text {GivTop }}$ have gone... There is no room in the car for the mothers, so they $y_{\text {GivTop }}$ run behind, and $\phi_{\text {GivTop }}$ lift up their trunks to avoid breathing the dust. The old Lady GivTop $_{\text {Gin }}$ is left alone. Sadly $\mathbf{N}_{\mathrm{New} F \mathrm{C}}$ she wonders: "When shall I see my little Babar again?" 
Alas, that very day, the king of the elephants SubTop has eaten a [a bad mushroom $]_{\text {NewTop }}$.

It $t_{\text {GivTop }}$ poisons him and he GivTop $_{\text {becomes }}$ ill, so ill that he

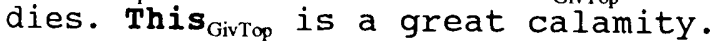

100 After the funeral the three oldest elephants are holding a meeting to choose a new King.

Just then they hear a noise. They ${ }_{\text {GivTop }}$ turn around. Guess what they see! \{Babar arriving in his car and all the elephants running and shouting: "Here NewFo $_{\text {they }}$ are!

105 Here $_{\text {NewFoc }}$ they are! Hello, Babar! Hello, Celeste! what beautiful clothes! what a beautiful car!" $\}_{\text {NewFoc }}$

Then [Cornelius, the oldest of all the elephants] NewTop $_{1}$ speaks in his quavering voice: "My good friends, we GivTop are seeking a King. Why not choose Babar? He $\mathbf{G i v T}_{\text {Top }}$ has just

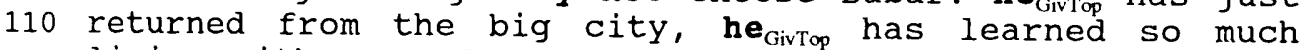
living with men, let us crown him King." All the other

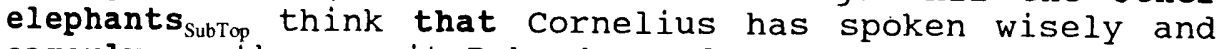
eagerly $y_{\text {NewFo }}$ they await Babar's reply.

$\left\{" I_{\text {GivTop }} \text { want to thank you one and all," }\right\}_{\text {NewFoc }}$ says

115 Babar, "but before accepting your proposal, $\mathbf{I}_{\text {GivTop }}$ must explain to you that, while we were traveling in the car, Celeste and I became engaged. If I become your King, she $_{\text {GivTop }}$ will be your Queen."

${\text { \{ } \text { Long }_{\text {NewFoc }} \text { live Queen Celeste! Long }}_{\text {NewFoc live King }}$ 120 Babar!" $\}_{\text {NewFoc }}$ cry all the elephants without a moment's hesitation. And thus EmphFoc it is that Babar becomes King.

$\left\{\text { "You } \text { GivTop }_{\text {have good ideas," }}\right\}_{\text {NewFoc }}$ says Babar to Cornelius, "I GivTop will therefore make you a general, and when I get my crown, $\mathbf{I}_{\text {GivTop }}$ will give you my hat. In a week NewFoc $_{\text {I shall }}$

125 marry Celeste. We GivTop will then have a splendid party in

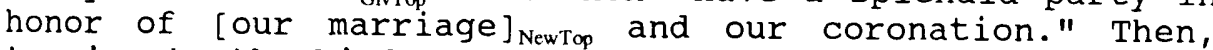
turning to the birds, Babar GivTop asks them to go and invite all the animals [to the festivities] NewTop, and he $_{\text {GivTop }}$ tells [the dromedary] $]_{\text {New Top }}$ to go to the town and buy [some

130 beautiful clothes] NewTop .

The wedding guests SubTop $_{\text {begin to arrive. The dromedary }}$ GivTop returns with the bridal costumes just in the nick of time.

After the wedding and the coronation everybody dances 135 merrily.

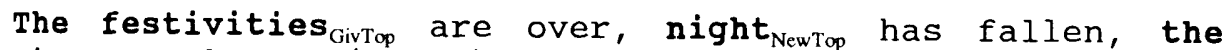
stars $_{\text {SubTop }}$ have risen in the sky. King Babar and Queen celeste GivTop $_{\text {are }}$ indeed very happy. 


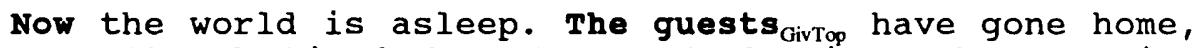

140 happy, though tired from too much dancing. They GivTop will long remember this great celebration.

And now King Babar and Queen Celeste, both eager for further adventures, set out on their honeymoon in a gorgeous yellow balloon.

\subsection{Commentary}

11. 1-4: In the great forest occupies $\mathrm{P} 1$ as a Locative satellite, and a little elephant occupies $\mathrm{s}$. In the great forest has been analysed as a NewTop, since the great forest is referred to in 11.74 and 85 ; a little elephant has also been treated as NewTop (ignoring the title) since the D-Topic "Babar the little elephant" regularly comes back in the later text.

A difficulty is that Dik (1989: 391) appears to exclude more than one NewTop per clause where he says "[t]he general rule will be that the NewTop constituent [our emphasis] captures the most prominent accent of the expression"; the first sentence constitutes a counterexample to any such exclusion.

His name and his mother are clearly subTops. She, in the final sentence, has been assigned GivTop on the assumption (for which no direct support can be found in Dik 1989, but see $n$. 6 above) that SubTops, which have "something "new"" about them (Dik 1989: 391), resemble NewTops in being able to "launch" GivTops. Finally, while occupies P1 as a subordinator.

one further issue arises, namely whether (with) her trunk is to be analysed as a NewTop, as a NewFoc, or as neither. Although it is inferrable, from elephant through mother, it is not subTop, since it is not in P1. NewTop is defensible on the basis of 1. 93, where the trunks of other mother elephants are referred to: in that sense, (with) her trunk introduces a D-Topic. If the distance between the two occurrences of trunk make this seem too far-fetched (but how is one to decide?), the possibility of NewFoc arises, yet there is no evidence here of special treatment, as would be necessary for NewFoc assignment. On balance, we see no reason to assign any pragmatic function to this term.

11. 5-7: Three GivTops, all in P1. In the last sentence, $\mathrm{P} 1$ is occupied by a verb, indicating an imperative 


\section{illocution.}

It is unclear how the assignment of ResTop function is to be operationalized. If some explicit indication of resumption is required (e.g. as I mentioned before, cf. Dik 1989: 277), then The story of Babar contains no ResTops. However, there is another operationalization thinkable, namely that ResTop is assigned whenever there has been a temporary interruption in a sequence of coreferential GivTops and the speaker/writer returns to that GivTop. Then the beginning of the story would be reanalysed as follows:

In the great forest $_{\text {NewTop }}$ a little elephant NewTop $_{\text {is born. }}$

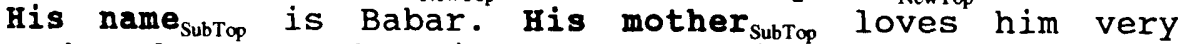
much. $\mathbf{s h e}_{\text {GivTop }}$ rocks him to sleep with her trunk while singing softly to him.

Babar $_{\text {ResTop }}$ has grown bigger. He $\mathbf{G}_{\text {GivTop }}$ now plays with ...

Whichever analysis is preferred, the choice, at least in the written language, appears to be without consequences.

11. 11-12: The assignment of ParFoc is justified by the parallelism of the three asyndetically coordinated clauses (in the sense of De Beaugrande and Dressler 1981: 57: "reusing surface formats but filling them with different expressions"). Note that the monkey and the birds also qualify for NewTop (by virtue of the monkeys in 1. 61-62 and the birds in 1. 127), and Babar of course for GivTop. It is unclear whether the assignment of ParFoc excludes or overrides the concomitant assignment of a Top function, or indeed vice versa. (Cf. the discussion of example (32) in $\$ 3$ above.)

11. 14-16: In the second sentence After several days, being followed by a comma, is taken to be in P2; very tired indeed, by contrast, is assumed to be a nonrestrictive modifier within $\mathrm{P} 1$ of he.

11. 17-18: We have noted $(\$ 2)$ that the relationship between the assignment of GivTop to a term and the givenness of the information carried by that term can be very indirect. This is particularly clear in these lines, where GivTop is assigned only to the first but not the equally given second occurrence of he; similarly, this, although given, cannot be GivTop, for P1 is occupied by 
because. In other words, GivTop cannot be assigned in syndetic embedded clauses, but can occur in asyndetic clauses.

11. 19-22: We have made two assumptions: that oneconstituent utterances have (only) P1 filled; that such P1-fillers may be marked for pragmatic functions. The former assumption follows from the requirement that $P 1$ be filled, a requirement that obtains for no other clausal position; the latter assumption follows from the use of P1 for "special purposes".

11. 23-25: some fine clothes has been assigned NewTop by virtue of coreferential anaphoric them in the next line, and the inferrable a fine suit in 1.28 .

Note (again; cf. 11. 1-4) that two Tops in one clause are possible, here one GivTop and one NewTop.

11. 26-30: As in 11. 17-18, the relation between givenness and GivTop is very indirect: only one of the five pronominal anaphoric references, she, counts as GivTop.

11. 31-38: In the third sentence, P1 is occupied by dummy it.

In 1. 35-36, the elevator boy is a SubTop. SubTops are defined as being inferred from a GivTop or NewTop. In this case, the term may, at a stretch, be inferred from (into) a big store (1. 31-32), a NewTop; yet inference from the elevator (1.32) seems more natural. Note, however, that this is not possible, since the elevator is neither Giv/SubTop (not being in P1) nor NewTop (being inferrable from a big store). Here too, we perceive givenness and Topic-assignment as being at odds.

In 1. 38, here is placed in P1 by virtue of NewFoc assignment (cf. The floorwalker $r_{\text {SubTop }}$ is here.)

11. 39-41: The colon after the object suggests particular saliency of the final constituent. Analysis as NewTop is encouraged by the reference to his purchases in 1.42 .

11. 42-43: Cf. the P1-filling in 1. 12 .

1. 44: Cf. the NewFoc assignment in 1. 38 .

11. 45-48: We assume that zero-anaphors, if in P1, carry 


\section{GivTop.}

The question arises whether he is correctly positioned in P1. We have assumed that it is preceded by two satellites in P2, separated from each other and from the clause proper by commas. If, however, we were to regard because he is tired as a parenthesis interrupting the sequence After dinner he goes to bed ..., P1 would then be occupied by After dinner and he could not be GivTop. Note that, whichever analysis is preferred (and there seems to be no way of establishing which is preferable), the linear positioning of he is unaffected. The assignment of GivTop is seen to be vacuous, an observation which we take to support our case for the superfluity of Topic assignment in English.

11. 52-54: for an automobile ride is a NewTop, launching the car.

11. 55-57: A learned professor is NewFoc rather than NewTop, since no overt reference is made to him in the subsequent discourse.

Comparing for an automobile ride NewTop $_{\text {in }} 1.52$ with a learned professor ${ }_{\mathrm{NewFo}}$ in 1. 55, we cannot but come to the conclusion that the distinction between the two functions is ill-founded: whereas the former barely introduces a Dtopic into the discourse (the availability of the car), the latter in fact functions as the starting-point for a five-predication paragraph about Babar's lessons, and thus clearly has a topic-introducing role. Nonetheless, the rules for NewTop and NewFoc impose upon the analyst the counter-intuitive assignments we have given.

11. 58-59: The problem pointed out for 11. 46-48 also applies here.

11. 65-69: One day during his walk is taken to be one constituent, and, not being separated off by a comma, to be in $\mathrm{P} 1$.

11. 70-71: some fine clothes is NewTop in anticipation of 1. 105-106. There is here also an element of Parfoc: cf. 1. $24 . .^{15}$

${ }^{15}$ Note that here, as well as in $11.33-35$ (ten times), there is parallelism, but no contrast. This 
11. 72-73: Strictly speaking the elephants have not been mentioned before, and as such qualify as NewTop; on the other hand, Babar and the other little elephants have been mentioned before, so that the totality of the elephants may be deduced by a bridging assumption (where there are young animals, there will also be adult animals), and qualify as subTop. For the status of bridging assumptions, see our comments on 11. 96-97.

11. 74-76: an old marabou bird is analysed as NewTop (although subTop might have been admissible here, given the birds in 1. 12). This is justified by the zeroanaphor in the second clause. The assumption that zeroanaphors, if in P1, carry GivTop here has consequences for the choice between NewTop and NewFoc status for the antecedent, since an old marabou bird is assigned NewTop despite the lack of any further overt reference to the DTopic in question; the covert reference, as it were, is made in the representation of the second co-ordinated clause.

11. 91-95: With Hannay (1985b), we assume that existential constructions introduced by There have no P1 position.

sadly is in $P 1$ position as a result of Focus assignment.

11. 96-97: The King of the elephants is a subset of the elephants and thus qualifies as a subTop. It is clear, however, that the notion of subTop needs more specification than is given in the open-ended list of relations proposed by Hannay (1985a: 53) and Dik (1989: 276). If the notion of "bridging assumption" is crucial to the definition of subTop, a reductio ad absurdum may be applied. Could it not be, say, that the monkey and the birds (11. 11-12), to some infinitesimal but real extent, can be linked back by bridging assumption to In the great forest, and therefore should be seen as subTops? can any term be excluded from bridging assumptions that are not restricted in some principled way?

suggests that, contrary to Dik's proposals, both contrastive and non-contrastive Focus elements may involve parallelism (see \$4.4). 
11. 98-99: This we take to be a GivTop referring back not to a first-order but to a second-order entity $\left(e_{\mathrm{i}}\right.$ : [die (he) ]). We assume that NewTop is assignable not only to terms but also to predications. For discussion, see $\$ 2.2$. above.

11. 100-101: See, on SubTops, the commentary on 11. 9697.

11. 102-106: The linguistic expression Babar ... car!" may be regarded as "all new" (cf. Hannay 1985b: $126 \mathrm{ff.}$ ), after the preceding Guess what they see!. As such, it receives the pragmatic function NewFoc and occupies P1.

11. 107-113: Compare eagerly with sadly in 1.94.

11. 119-121: According to Dik (1989: 282), ContrFoc is either Parallel or Counter-presuppositional. As suggested in $\$ 2.3$ above, we believe it is necessary also to recognize cases where there is neither parallelism nor negation of presuppositions, but there is salience. One such case is thus in 1 . 121, which we mark, following Hannay (1983: 210), as Emph(atic) Foc.

11. 122-130: Note that our marriage is here NewTop by virtue of later reference to the wedding guests (1. 131).

11. 136-138: There is an alternative analysis of the first sentence with three cases of ParFoc (of the type carried out in 11. 11-12). Such an assignment might well affect the associated prosodic contours, but is without consequences in the written language.

\subsection{Conclusions}

The first conclusion to be drawn from the analysis in $\$ 4.2$, and the commentary in $\$ 4.3$, is that the analysis is possible. Given the principles of analysis detailed in \$4.1, the proposals for pragmatic function assignment and the rules for P1-placement of constituents can be operationalized. We hope and intend that our experience with The story of Babar, as protocolled in our assignments and in our commentary, will be of use to other linguists wishing to employ the theory of FG in the analysis of discourse. 
Although we have shown that current proposals can indeed be put into practice, we may also raise questions as to the insightfulness of the resultant analysis. Let us take each of the pragmatic functions in turn.

All 82 instances of GivTop also receive the syntactic function subj.

Similarly, of the 11 instances of subTop, 9 are subj; in the 2 two remaining cases $(11,19-20)$, the subTop is the only constituent and therefore can only appear in P1. Again, P1-placement of subj would constitute a sufficient rule.

There are no ResTops (pace the commentary on 11. 5-7, but, there too, the would-be ResTop is subj of its clause.).

We take these observations to support our proposal in $\$ 3$ to do away with Giv/Sub/Res Topic assignment in English and to make P1, if not filled by a prior claimant, a home for subj. The question arises whether the combination obj(Giv/Sub/Res)Top occurs in P1 in English, for then P1-placement would be attributable to the Topic assignment and the case for Topic would be restored. Clearly, this is an empirical matter, but our hypothesis is that the combination of Obj, Top and P1 is unknown in English. All constructed examples of obj in P1 seem to have Foc rather than Top function:

(33) A: Now that you're back from Paris, can I ask you something? People react differently to different sights in Paris. What about the Eiffel Tower?

B: The Eiffel Tower I rather liked. (modified from Dik 1978: 143)

The Eiffel Tower does seem to pick up the NewTop in A's question, and therefore could qualify as objTop. Nevertheless we suspect that The Eiffel Tower will be associated with the "pronounced prosodic pattern" reserved for ParFoc constituents and will regularly be followed by such continuations as but the Pompidou centre I was crazy about. We further suspect that if the speaker intends no contrast with any other Parisian sight, she will tend to construe The Eiffel Tower as Subj-in-P1, e.g. The Eiffel Tower was one of my favourites. This issue clearly deserves attention in future research.

Sentences containing two Focus constituents also seem to present a problem. Consider, for instance, the 
following variation on (33):

(33') A: Now that you're all back from Paris, can I ask you something? People react differently to different sights in Paris. What sights did the various people like?

B: Well, The Eiffel Tower John rather liked, The Pompidou Centre Mary liked best, etc.

The problem will be clear: if two constituents (e.g. The Eiffel Tower and John) are both (Parallel) Focus, then how do we decide which goes into P1? For the present we will assume that in such cases the Focus constituent with the highest degree of topicality (or "aboutness") will go into P1. In (33') the constituents The Eiffel Tower and The Pompidou Centre can be considered more topical than John and Mary on account of the fact that in the preceding question it is what sights that is placed in P1 position. Since sentences may contain two, or more, Focus constituents, and since Focus constituents can be given special treatment in a number of ways, further research will have to establish on which grounds a particular form of special treatment is given to a particular focus constituent.

We identified 20 instances of NewTop in the text, but failed to find in the written text any formal correlation between NewTop assignment and the placement of constituents so marked. Whereas Dik (1989: 269) claims that NewTops have a strong preference for taking late (or at least non-initial) position in the clause, this is not supported by the analysis of The story of Babar. Of the 20 NewTops, 7 appear in final $X$ position (i.e. after 0 ), but 4 in 0,2 in $S$, and again 7 in $P 1$. We take this finding to support our proposal in $\$ 3$ to regard those instances of NewTop where there is some expression correlate of the function assignment (positionally or prosodically) as a sub-type of Focus, namely presentative Focus. All positionally or prosodically undistinguished instances will be analysed as receiving no pragmatic function at all.

There are 15 instances of NewFoc in our analysis. These all occur in P1. 9 are associated with the secondary functional pattern for English, P1 Vf S Vi O (Dik 1978: 185), 5 with the primary pattern, and 1 constitutes a full expression. FOC is assigned to instances of subj, Obj, Loc, Man, and Time: these differential assignments 
justify the retention of the pragmatic function Focus.

There is 1 instance of EmphFoc; it occurs in P1. (For discussion, see $\$ 4.3$, commentary on 11. 119-121, above.)

There are 7 , possibly 10 (cf. $\$ 4.3 .$, commentary on 11 . 136-138) instances of Parfoc. These occur in various positions; the justification for assigning the pragmatic function to the constituents in question is derived from their participation in parallelism (in the sense of De Beaugrande and Dressler referred to in $\$ 4.3$ above, 11 . 11-12).

For the reasons stated in $\$ 3$, we are of the opinion that, unlike Topic, Focus is relevant in English. However, in order to render Focus function applicable, we propose a classification of Focus according to (at least) the following four dimensions:

- completive (contextually New) vs non-completive (contextually Given)

- presentative vs non-presentative

- emphatic vs non-emphatic

- contrastive vs non-contrastive

The classification we have in mind can be represented as follows: ${ }^{16}$

${ }^{16}$ The classification in Figure 2 presents all possible subtypes of Focus. This does not necessarily mean that all these types are given special treatment in every language. It may, in fact, be an interesting research question to establish which types of Focus are given special treatment in different languages.

As will be clear, the specification of the subtypes of Focus could be abbreviated by means of redundancy rules:

$$
\begin{array}{lll} 
\pm \text { Pres } & ----> & + \text { Compl } \\
\text {-Compl } & ---> & + \text { Emph } \\
\pm \text { Con } & ----> & + \text { Emph }
\end{array}
$$


Figure 2

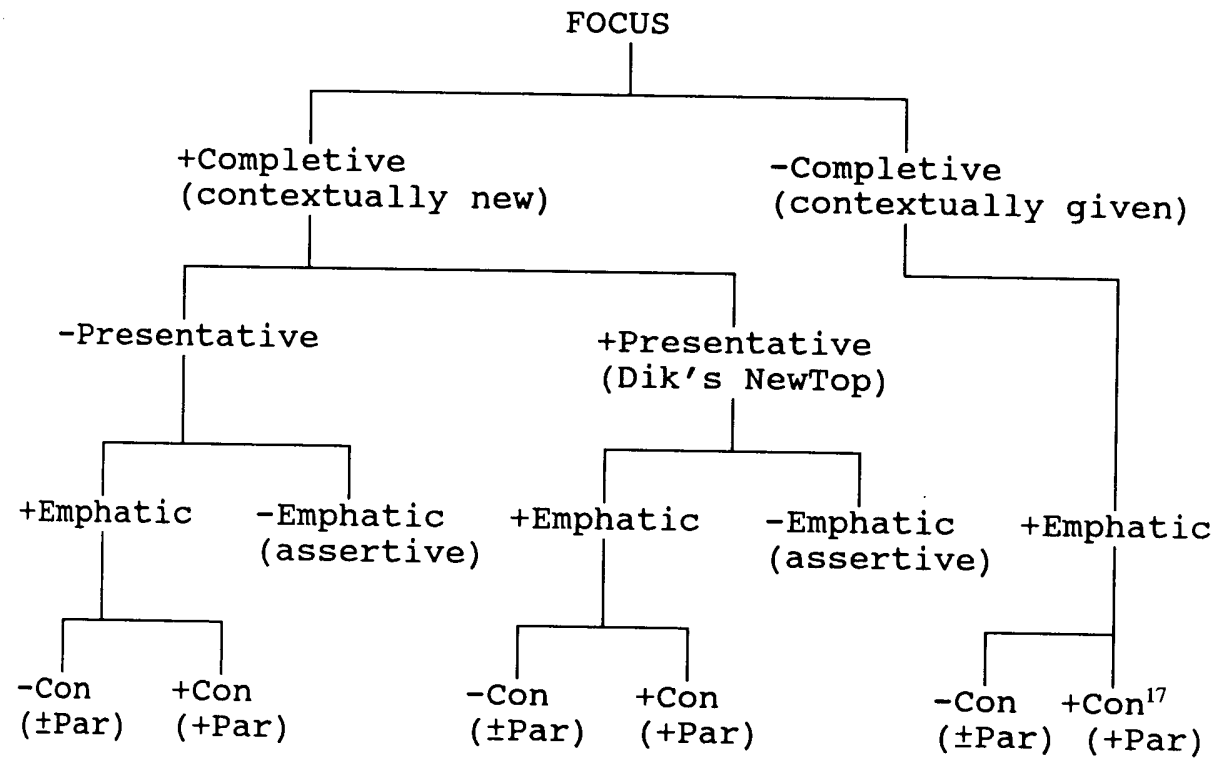

Examples:

(34) $\mathrm{X}$ : Where is John going?

$\mathrm{Y}$ : John is going to the market (+Compl -Pres -Emph Focus, cf. (7))

(35) John gave a party last week, but the music was awful. (+Compl +Pres -Emph Focus, cf. (4))

(36) John bought a car, of all things. (+Compl -Pres + Emph -Con Focus, cf. (i), n. 8)

${ }^{17}$ According to this classification, -Compl +Emp +Contr Focus (which always involves an element of parallel) covers both Dik's parallel Focus and his Counterpresuppositional Focus, the reason being that, in our view, all instances of counterpresuppositional Focus (of which we did not find any examples in the text, but see examples (9)-(12)) involve parallelism. 
(37) It is such fun to ride up and down in this funny box, that he rides all the way up ten times and all the way down ten times. (+Compl -Pres +Emph +Con Focus, see also (28))

(38) Suddenly, right before our eyes, there appeared a huge elephant. (+Compl +Pres +Emph -Con Focus, cf. (2))

(39) The monkey hides, the birds fly away, Babar cries. (+Compl +Pres +Emph +Con Focus, see 11. 9-11 of The story of Babar and our commentary)

(40) A: What about Rebecca?

B: It was to her that John gave his most precious painting. (-Compl +Emph -Con Focus, cf. (31))

(41) John and Bill came to see me. John was nice, but Bill was rather boring. (-Compl +Emph +Con Focus, cf. (32))

Summarizing, we conclude that the analysis of The story of Babar provides evidence for:

(a) abolishing GivTop, ResTop and SubTop as pragmatic functions relevant to English;

(b) redefining NewTop as a subtype of Focus, relevant only where there is some expression correlate (= special treatment);

(c) retaining Focus assignment in English, subclassifying it as in Figure 2 above.

\section{Epilogue}

The purpose of this paper has been to explore the consequences of the proposals made by Dik (1989) for the assignment of pragmatic functions. We have attempted to clarify the double assault on this problem undertaken by FG and to indicate ways in which the two approaches can connect. We have examined in detail the partial correspondence of the Given-New and the Topic-Focus distinctions, concluding that the hybrid pragmatic functions that result from their combination lead to problems and inconsistencies. Turning to English, we have suggested that Topic assignment is superfluous, but Focus assignment is necessary, albeit differently subclassified. We have applied Dik's (1989) proposals to a text, concluding that they can be operationalized, but that they yield an analysis that suffers from a number of 
inconsistencies and unclarities.

our alternative may perhaps best be summarized by proposing a set of ordered expression rules for the P1placement of constituents in English:

(R1) If a clause consists of one constituent only, this constituent must be placed in P1.

(R2) If a clause consists of more than one constituent, one of which is a P1-constituent (question word, subordinator, relative pronoun), place this P1constituent in P1.

(R3) Else, if a clause is interrogative (yes-no question) or imperative, ${ }^{18}$ place $V_{f}$ in $\mathrm{P} 1$.

(R4) Else, if a clause contains a constituent with Focus function, place this Focus constituent in P1. (Alternatively, Focus constituents may be given special treatment by means of prosodic prominence, parallelism, or any combination of these focalizing devices).

(R5) Else, place a $\sigma_{2}$ - or $\sigma_{3}$-satellite (i.e. level 2 or 3 satellite, cf. Dik (1989: 206)) in P1 (optional).

(R6) Else, place constituent with subject function (including dummy it) in P1 (unmarked case).

${ }^{18}$ In the case of imperative sentences it is sometimes possible for a satellite to appear in P1 (e.g. now, simply, just). Note, however, that this possibility seems to be restricted to a very small group of typically oneword satellites (or perhaps even operators) with a mitigating function. 


\section{REFERENCES}

Beaugrande, R.-A. de and W.U. Dressler Introduction to text linguistics. London: Longman.

(1981)

Brown, G. and G. Yule (1983) Discourse analysis. Cambridge: CUP.

Carpenter, P.A. and M.A. Just (1977) Integrative processes in comprehension. In D. LaBerge \& S.J. Samuels (eds.), Basic processes in reading: perception and comprehension. Hillsdale, NJ: Lawrence Erlbaum, 217-241.

Chafe, W.L. (1976) Givenness, contrastiveness, definiteness, subjects, topics and points of view. In C.N. Li (ed.), subject and topic. New York: Academic Press, 25-57.

Clark, H.H. and S.E. Haviland (1977) Comprehension and the Given-New contract. In R.O. Freedle (ed.), Discourse production and comprehension. Hillsdale NJ: Lawrence Erlbaum, 1-40.

Cruse, D.A. (1980) Review of Hawkins (1978), Definiteness and indefiniteness. Journal of Linguistics 16: 308-316.

Dik, S.C. (1978) Functional Grammar. Amsterdam: NorthHolland.

Dik, S.C. (1989) The theory of Functional Grammar. Part 1. Dordrecht: Foris.

Dik, S.C. (forthcoming) The theory of Functional Grammar. Part 2.

Ebert, K.H. (1971) Referenz, Sprechsituation und die bestimmten Artikel in einem nordfriesischen Dialekt (Fering) . Bräist/Bredstedt: Nordfriisk Instituut.

Groot, C. de (1981) Sentence intertwining in Hungarian. In A.M. Bolkestein, H.A. Combé, S.C. Dik, C. de Groot, J. Gvozdanovic, A. Rijksbaron \& C. Vet (eds.), Predication and expression in Functional Grammar. London: Academic Press, 41-62. 
Halliday, M.A.K. (1985) An introduction to Functional Grammar. London: Arnold.

Halliday, M.A.K. and R. Hasan (1976) Cohesion in English. London: Longman.

Hannay, M. (1983) The Focus function in Functional Grammar: questions of contrast and context. In S.C. Dik (ed.), Advances in Functional Grammar. Dordrecht: Foris, 207-223.

Hannay, M. (1985a) Inferrability, discourse-boundness, and sub-topics. In A.M. Bolkestein, C. de Groot \& J.L. Mackenzie (eds.), syntax and pragmatics in Functional Grammar. Dordrecht: Foris, 49-63.

Hannay, M. (1985b) English existentials in Functional Grammar. Dordrecht: Foris.

Hannay, M. (1990) Pragmatic function assignment and word order variation in a Functional Grammar of English. Working Papers in Functional Grammar 38. To appear in Journal of Pragmatics.

Jong, J.R. de (1981) On the treatment of Focus phenomena in Functional Grammar. In T. Hoekstra, H. van der Hulst \& M. Moortgat (eds.), Perspectives on Functional Grammar. Dordrecht: Foris, 89-115.

Löbner, S. (1985) Definites. Journal of Semantics 4: 279326.

Prince, E.F. (1981) Toward a taxonomy of Given-New information. In P. Cole (ed.), Radical Pragmatics. New York: Academic Press, 223-255.

Sanford, A.J. and S.C. Garrod (1981) Understanding written language: explorations of comprehension beyond the sentence. Chichester: Wiley. 